\title{
A novel in silico reverse-transcriptomics-based identification and blood-based validation of a panel of sub-type specific biomarkers in lung cancer
}

Debmalya Barh ${ }^{1 *}$, Neha Jain ${ }^{1,2}$, Sandeep Tiwari ${ }^{1}$, John K Field ${ }^{3}$, Elena Padin-Iruegas ${ }^{4}$, Alvaro Ruibal ${ }^{5}$, Rafael López ${ }^{4}$, Michel Herranz ${ }^{6}$, Antaripa Bhattacharya ${ }^{1}$, Lucky Juneja ${ }^{1,2}$, Cedric Viero $^{1,7}$, Artur Silva ${ }^{8}$, Anderson Miyoshi ${ }^{9}$, Anil Kumar ${ }^{2}$, Kenneth Blum ${ }^{1,10}$, Vasco Azevedo ${ }^{9}$, Preetam Ghosh ${ }^{1,11}$, Triantafillos Liloglou ${ }^{3}$

From 8th International Conference of the Brazilian Association for Bioinformatics and Computational Biology (X-meeting 2012)

Campinas, Brazil. 14-17 October 2012

\begin{abstract}
Lung cancer accounts for the highest number of cancer-related deaths worldwide. Early diagnosis significantly increases the disease-free survival rate and a large amount of effort has been expended in screening trials and the development of early molecular diagnostics. However, a gold standard diagnostic strategy is not yet available. Here, based on miRNA expression profile in lung cancer and using a novel in silico reverse-transcriptomics approach, followed by analysis of the interactome; we have identified potential transcription factor (TF) markers that would facilitate diagnosis of subtype specific lung cancer. A subset of seven TF markers has been used in a microarray screen and was then validated by blood-based QPCR using stage-II and IV non-small cell lung carcinomas (NSCLC). Our results suggest that overexpression of HMGA1, E2F6, IRF1, and TFDP1 and downregulation or no expression of SUV39H1, RBL1, and HNRPD in blood is suitable for diagnosis of lung adenocarcinoma and squamous cell carcinoma sub-types of NSCLC. Here, E2F6 was, for the first time, found to be upregulated in NSCLC blood samples. The miRNA-TF-miRNA interaction based molecular mechanisms of these seven markers in NSCLC revealed that HMGA1 and TFDP1 play vital roles in lung cancer tumorigenesis. The strategy developed in this work is applicable to any other cancer or disease and can assist in the identification of potential biomarkers.
\end{abstract}

\section{Introduction}

Lung cancer is the leading cause among cancer related deaths worldwide, constituting $17 \%$ of new cancer cases and $23 \%$ of deaths from cancer. Although N. American and European countries show a slow decline in death rates due to lung cancer, deaths due to this form of cancer are increasing considerably in Asian and African countries [1]. Lung cancer is mainly divided into two

\footnotetext{
* Correspondence: dr.barh@gmail.com

'Centre for Genomics and Applied Gene Technology, Institute of Integrative Omics and Applied Biotechnology (IIOAB), Nonakuri, Purba Medinipur, WB721172, India

Full list of author information is available at the end of the article
}

subtypes, small cell lung cancer (SCLC), which accounts for 10-15\% of all cases and non-small cell lung cancer (NSCLC, 85-90\%). The latter group is further histologically subdivided into four categories; adenocarcinoma, squamous cell carcinoma, large cell carcinoma and 'others', for example cancers of neuroendocrine origin [2]. The overall 5-year survival rate for NSCLC ranges from $9 \%$ to $15 \%$ [3]. The high mortality from lung cancer is due a combination of lack of reliable early diagnostic tools $[3,4]$ along with a poor arsenal of lung cancer regimens for stage I lung cancer, whose survival rate is also surprisingly low [5].

\section{() Biomed Central}

(c) 2013 Barh et al.; licensee BioMed Central Ltd. This is an open access article distributed under the terms of the Creative Commons Attribution License (http://creativecommons.org/licenses/by/2.0), which permits unrestricted use, distribution, and reproduction in any medium, provided the original work is properly cited. 
Numerous studies have utilized different "-omics"based approaches to identify molecular signatures in lung cancer with diagnostic or prognostic value while using minimally invasive processes. Some of these are as follows: 34 miRNA signatures [6], expression profiles of 11 miRNAs (miR-106a, miR-15b, miR-27b, miR-142-3p, miR-26b, miR-182, miR-126, let7g, let-7i and miR-30e5p) from serum [7], 7 miRNA signatures [8], overexpression of six snoRNAs [9], and expression of $3 \mathrm{miRs}$ (miR-205, miR-210 and miR-708) in sputum [10]. Additional signatures and markers have also been reported from the plasma proteome $[11,12]$, the salivary proteome [13], the serum epigenome [14], sputum-based genomics [15], and blood-based gene expression studies [16]. However, none of these have progressed sufficiently to provide the necessary specificity and sensitivity required for clinical implementation.

microRNAs (miRNAs/miRs) are involved in a variety of biological processes, including cell cycle regulation, cell differentiation, development, metabolism, and aging [17]. They have also been shown to be aberrantly expressed in several cancers [18]. Lung cancer is no exception to this and miRNA signatures have been suggested to be useful in diagnosis, prognosis, and therapy [7,19-21]. miRNAs regulate posttranscriptional gene expression and a single miRNA can regulate up to 200 mRNAs including those for transcription factors (TFs) [22]. Because miRNA transcription is under the regulation of TFs, intriguing feedback and feed-forward regulatory loops can be formed among TFs and miRNAs [17].

In this study we have developed a novel in silico reverse-transcriptomics strategy followed by interactome analysis to identify the sub-type specific diagnostic TF markers in lung cancer. The approach is novel as the sub-type specific TF markers were identified starting with experimentally validated miRNA profiles in lung cancer. We have also attempted to provide a molecular insight during the early events in lung cancer.

\section{Materials and methods}

\section{Literature mining}

Extensive literature and text mining was carried out to collect deregulated miRNAs in lung cancers (NSCLC and SCLC) using databases such as PubMed, Sirus, and Elsevier as well as search engines such as Google and Google Scholar. miR2Disease [23] was also used to gather lung cancer specific miRNAs information. Priority was given to reports that have used markers based on biopsy samples and patient's remote media (blood, serum, plasma, sputum, and bronchioalveolar lavage among others [24]). Selected miRNAs were then grouped into three categories: (1) NSCLC specific, (2) exclusively SCLC related, and (3) common in both the types. The up- and down-regulated
miRNAs within each of these three groups were also noted.

\section{GO assignment to miRNAs using reverse annotation strategy}

No tool is currently available to classify or cluster miRNAs as per their GO (Gene Ontology) or functional annotation. We applied a reverse approach in which GO terms to a miRNA are assigned based on the functional annotation of the targets of the particular miRNA. In this approach, we first identified experimentally validated targets of each miRNA using miRNA target databases miRWalk [25], miRecords [26], miReg [17], and miRTarBase [27]. Next, targets for each miRNA were subjected to ToppGene Suite [28] for GSEA (Gene Set Enrichment Analysis) candidate gene prioritization. The top-ranked genes were used in DAVID v6.7 [29] analysis for functional annotation clustering and the assignment of GO terms to each miRNA which targets these genes. GO terms related to various aspects of cancer were considered. miRNAs and their corresponding targets that fall under these specific GO categories were selected, and the rest were ignored (Figure 1, Step-3).

\section{miRNA-TF-miRNA or TF-miRNA-TF interactions}

To date, there is no study reporting direct miRNAmiRNA interaction. However, it is well known that miRNAs can modulate post-transcriptional gene regulation as well as their own expression through feed-back and feed-forward loops that are mediated by various TFs. Therefore, there are miRNA-TF interactions. As TFs interact with other TFs and proteins, the known TF-TF networks can be complemented by integrating the relevant miRNA-TF interactions to make TF-miRNA-TF or TF-miRNA-TF-miRNA interactions. Such TF-miRNATF-miRNA interaction networks will indirectly represent the miRNA-miRNA interactions.

We thus created a cancer specific TF-TF interaction network using targets of miRNAs frequently deregulated in NSCLC, SCLC, or common to both of these types utilizing Osprey v1.0.1 [30] (Figure 1, Step-3). To achieve this, we selected all experimentally validated, highly ranked miRNA targets of NSCLC, SCLC, or common to both that were identified in the previous step and fed them into Osprey (Figure 1, Step-6). The protein-protein interaction (PPI) network for each cancer type generated by Osprey was first filtered sequentially with the "Transcription", "Cell cycle" and "Cell cycle biogenesis" GO filters in Osprey (Figure 1, Step-8). Therefore, the resultant TF-TF interaction network is cell cycle specific. The sequential filters were used because cell cycle deregulation is one of the major BPs (Biological Processes) that is affected during tumorigenesis. 


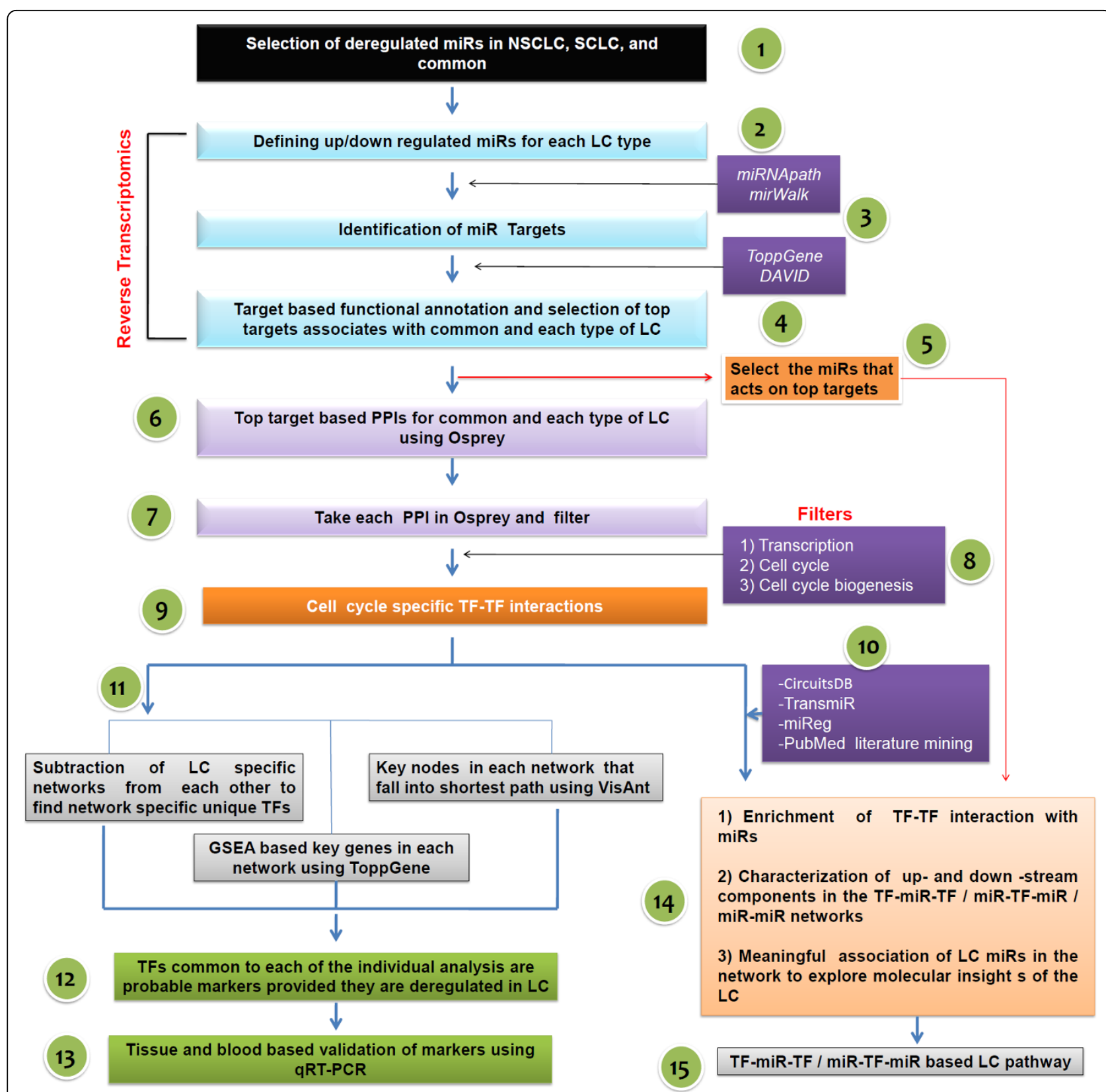

Figure 1 Flow-diagram showing entire strategy that is applied to identify TF biomarkers in Lung cancer based on miRNA profiles.

This cell cycle specific TF-TF network was further enriched by manually mapping the interacting miRNAs with data collected from the miReg [17], TransmiR [31], and CircuitsDB [32] databases and from literature mining to create a TF-miRNA-TF interaction map (Figure 1, Step-10). Because we have selected lung cancer related miRNAs (based on GO assignment in the previous step) and developed a network using their targets, this network represents the interaction of TFs involved in lung cancer tumorigenesis. Based on our earlier hypothesis, this interaction map also represents the miRNA-TF-miRNA or TF-
miRNA-TF interaction map that is common to both NSCLC and SCLC. Similarly, NSCLC and SCLC specific miRNA-TF-miRNA or TF-miRNA-TF or miRNA-miRNA interaction maps were created using targets of NSCLC and SCLC unique miRNAs. Therefore, a total of three networks were generated (Figure 1, Steps-14-15).

\section{Marker identification}

The miRNA-TF-miRNA or TF-miRNA-TF interaction maps for NSCLC, SCLC, and common developed in the previous steps were analyzed by subtracting from each 
other to identify the NSCLC, SCLC, and a common pathway that is specific unique TFs. Each network was further analyzed using the protein-protein interaction (PPI) analysis tool VisANT [33] to identify the key nodes and the shortest cancer specific pathways in each network. Key nodes in a PPI network are identified as having the highest number of interactions. Therefore, such key node proteins are often involved in multiple signaling pathways, and if a key node protein falls in a shortest path, the node might be treated as a marker of a disease provided that its expression is altered in that disease state. In the third strategy, we utilized GSEA identification of key genes in each network using ToppGene Suite [28]. When all of the data from each of these three analyses had been obtained, we identified the TFs common to each of the individual analyses (Figure 1, Steps-11-12). Therefore, these sets of common TFs were putative markers, and the TFs that were a part of NSCLC network could be treated as a NSCLCspecific marker.

\section{Experimental validation of markers}

Once we had selected the potential markers, we checked their expression levels initially in lung cancer tissue samples using microarrays and then further validated them using patient's blood samples and quantitative RTPCR (qPCR) (Figure 1, Step-13).

\section{Interrogation of data from expression microarray}

The frozen tissue samples examined from 30 squamous cell carcinomas and 30 adenocarcinomas (each is a type of NSCLC) from the Liverpool Lung Project tissue bank. All samples were of pathological stage T2. RNA was extracted using the RNeasy kit (Qiagen). Five RNA pools from five adjacent normal lung tissues were also profiled for comparison purposes. The microarray experiments were performed by Almac (Belfast, UK). Total RNA was amplified using the NuGENTM Ovation $^{\text {TM }}$ RNA Amplification System V2. First-strand synthesis of cDNA was performed using a unique firststrand DNA/RNA chimeric primer mix, resulting in cDNA/mRNA hybrid molecules. Following fragmentation of the mRNA component of the cDNA/mRNA molecules, second-strand synthesis was performed, and double-stranded cDNA was produced with a unique DNA/RNA heteroduplex at one end. In the final amplification step, RNA within the heteroduplex was degraded using $\mathrm{RNaseH}$, and a replication of the resultant singlestranded cDNA was achieved using the DNA/RNA chimeric primer binding and DNA polymerase enzymatic activity. The amplified single-stranded cDNA was purified to allow accurate quantitation of the cDNA and to ensure optimal performance during the fragmentation and labeling process. The single-stranded cDNA was assessed using spectrophotometric methods in combination with the Agilent Bioanalyzer.

The appropriate amount of amplified single-stranded cDNA was fragmented and labeled using the FL-Ovation ${ }^{\mathrm{TM}}$ cDNA Biotin Module V2. The enzymatically and chemically fragmented product (50-100 nt) was labeled via the attachment of biotinylated nucleotides onto the 3'-end of the fragmented cDNA.

The resultant fragmented and labeled cDNA was added to the hybridization cocktail in accordance with the NuGENTM guidelines for hybridization onto Affymetrix GeneChip ${ }^{\circledR}$ arrays. Following hybridization for 16-18 hours at $45^{\circ} \mathrm{C}$ in an Affymetrix GeneChip ${ }^{\circledR}$ Hybridization Oven 640 , the array was washed and stained on the GeneChip ${ }^{\circledR}$ Fluidics Station 450 using the appropriate fluidics script and then inserted into the Affymetrix autoloader carousel and scanned using the GeneChip ${ }^{\circledR}$ Scanner 3000.

The Rosetta Error Model has been applied to the raw data to generate the processed data. The profile comparisons between cancerous lesions and normal RNA pools utilized Student's t-test. The Benjamini \& Hochberg multiple test correction method was also employed.

\section{Validation using quantitative RT-PCR ( $q P C R$ )}

Blood samples, RNA isolation, and $c D N A$ preparation As our focus is NSCLC, blood samples from 8 metastatic lung adenocarcinoma, 8 metastatic squamous cell lung carcinoma patients, and 5 healthy volunteers (control) were used for the validation. Patient eligibility criteria were as follows: 18 years of age or older, in clinical stage II-IV based on the International TNM classification, performance status of 0 to 2, and no other malignances. All patients and volunteers have signed informed consent forms. Ten milliliters of EDTA blood sample was collected from the selected groups before chemotherapy treatment. Blood samples were centrifuged at $2000 \mathrm{~g}$ for $10 \mathrm{~min}$ and the serum phase was separated and frozen at $-80^{\circ} \mathrm{C}$. The Buffy Coat (white blood cells and circulating tumor cells) was collected and processed by lysis (Ammonium Chloride, TRIS, $\mathrm{ddH}_{2} \mathrm{O}$ ) and then washed with PBS. The dry pellet was kept at $-80^{\circ} \mathrm{C}$ until RNA isolation. RNA was purified by Quiamp RNA Blood Mini Kit (QIAGEN Inc., USA) according to the manufacturer's instructions. cDNA was synthesized with random hexamer primers (Deoxynucleoside Triphosphate set, Roche, Germany) at $10 \mathrm{mM}, \mathrm{MgCl}_{2}, \mathrm{MuLV}$ Reverse Transcriptase, PCR Buffer, RNAse Inhibitor, and random hexamers from Applied Biosystems USA. The resulting cDNA was stored at $-20^{\circ} \mathrm{C}$ until further use.

\section{Quantitative RT-PCR (qPCR)}

qPCR was carried out using SYBR $^{\circledR}$ Green Master Mix (Applied Byosistems, USA) and Applied Biosystem's 7500 real-time PCR system according to the manufacturer's instructions. Primers for GAPDH were designed 
with Vector NTI Advance ${ }^{\mathrm{TM}} 11$ (Invitrogen) and primers for TFDP1, SUV39H1, RBL1, E2FG, IRF1, HMGA1, and HNRPD were designed using qPrimerDepot (http://primerdepot.nci.nih.gov/). To avoid the influence of genomic contamination, the amplicons spanned at least one intron. The primers used are listed in Additional file 1. qPCR was performed in a final volume of $20 \mu \mathrm{l}$ with a SYBR PCR Master Mix, using $1 \mu \mathrm{l}$ cDNA. Cycling conditions were $95^{\circ} \mathrm{C}$ for $10 \mathrm{~min}$, followed by 40 cycles at $95^{\circ} \mathrm{C}$ for $15 \mathrm{~s}$ and $60^{\circ} \mathrm{C}$ for $1 \mathrm{~min}$ each to obtain the melting curve.

Relative gene expression levels were determined by the quantitative curve method. Quantitative normalization of the cDNA in each sample was performed using GAPDH gene expression as an internal control. Target gene mRNA levels were given as ratios to GAPDH mRNA levels. qPCR assays were performed in duplicate for each sample, and the mean value was used to calculate the mRNA expression levels.

\section{Results}

\section{miRNA statistics in lung cancer}

We selected 184 miRNAs for NSCLC and 62 for SCLC using literature mining and the miR2 Disease database. Among these 246 miRNAs, 41 were found to be involved in both of the lung cancers and therefore are common miRNAs involved in lung cancer regardless of the subtype (Figure 1, Step-1). In the common miRNA group, 13 and 11 miRNAs were found to be up- and downregulated, respectively; whereas 18 miRNAs showed differential expression, i.e., either upregulated in SCLC and downregulated in NSCLC or vice versa (Figure 1, Step-2) (Additional file 2). A total of 22 miRNAs were found to be unique to SCLC (16 upregulated and 6 downregulated) (Additional file 3). For NSCLC, the total number of unique miRNAs was 143, (89 upregulated and 43 downregulated) (Additional file 4).

\section{Target-based functional annotation of miRNAs}

Using miRWalK, miRBASE, miRecord, miRTarBASE, and miReg we identified several validated targets for each miRNA. Thereafter, as per our reverse transcriptomics strategy, targets for each miRNA were subjected to gene enrichment analysis using ToppGene Suite as described in Materials and Methods (Figure 1, Step-3). Top targets that are associated with common, NSCLC, and SCLC were identified. DAVID-based functional annotations of the top targets revealed that most of these targets are cell cycle related, so the miRNAs that have these targets are related to transcription, cell cycle regulation, cell biogenesis and organization, cell proliferation, and other biological processes related to tumorigenesis. The list of common miRNAs involved in lung cancer along with their corresponding GO terms is presented in Additional file 5. miRNAs involved uniquely in either NSCLC or SCLC and their corresponding GO terms were also defined (data not shown).

\section{miRNA-miRNA interaction network in lung cancer Interaction of common miRNAs}

Based on the hypothesis that interactions of miRNA-TFmiRNA or TF-miRNA-TF-miRNA targets represent miRNA-miRNA interactions, we used gene enrichment based on the top targets of miRNAs common to NSCLC and SCLC in Osprey to create a protein-protein interaction map (Figure 1, Steps-6-7). In total, 638 targets corresponding to 40 common miRNAs generated a map having 1791 nodes in Osprey. Keeping in mind that miRNA genes are regulated by transcription factors (TF), miRNAs regulate TFs, and, as the gene enrichment analysis shows, most of the miRNAs regulate transcription, the network of 1791 nodes is filtered with the "Transcription factor" filter in Osprey and subsequently only 170 nodes are retained. This transcription network of 170 nodes is further filtered with "Cell cycle" and "Cell Organization and Biogenesis" filters, as per the enriched GO categories (Figure 1, Step-8), and finally the cell cycle specific total of 26 key TF nodes in common events, NSCLC, and SCLC are found (Figure 1, Step-9 and Figure 2).

\section{Interactions of SCLC associated miRNAs}

For SCLC, 634 nodes are used in total to create the interaction map in Osprey. The resultant map is sequentially filtered with "transcription factor", "Cell cycle", and "Cell organization and biogenesis" Filters and only 9 key nodes are obtained (Figure 1, Steps-6-9 and Figure 3).

\section{Interactions of NSCLC linked miRNAs}

Similar methods of network creation and filtering to those applied to identify key nodes in common and in SCLC (Figure 1, Steps-6-9) were adopted to generate a key interaction network in NSCLC. A total of 2421 nodes are filtered and finally 27 nodes are obtained (Figure 4).

\section{SCLC network is a part of NSCLC}

Next we subtracted the LC specific networks from each other to identify unique network specific TFs (Figure 1, Step-11). In the 27 nodes of the NSCLC network (Figure 4), all of the SCLC nodes (Figure 2) are found to be present (Figure 4, in red circle). Therefore, it is evident that there are additional pathways involved in NSCLC compared to SCLC and the SCLC network represents a subset of the NSCLC network.

\section{Genes involved in common events in lung cancer}

Next, we compared the common network (Figure 2) with the SCLC (Figure 3) and NSCLC and SCLC networks (Figure 4) by subtracting each from the other to identify key nodes that are common to (1) SCLC and NSCLC; 


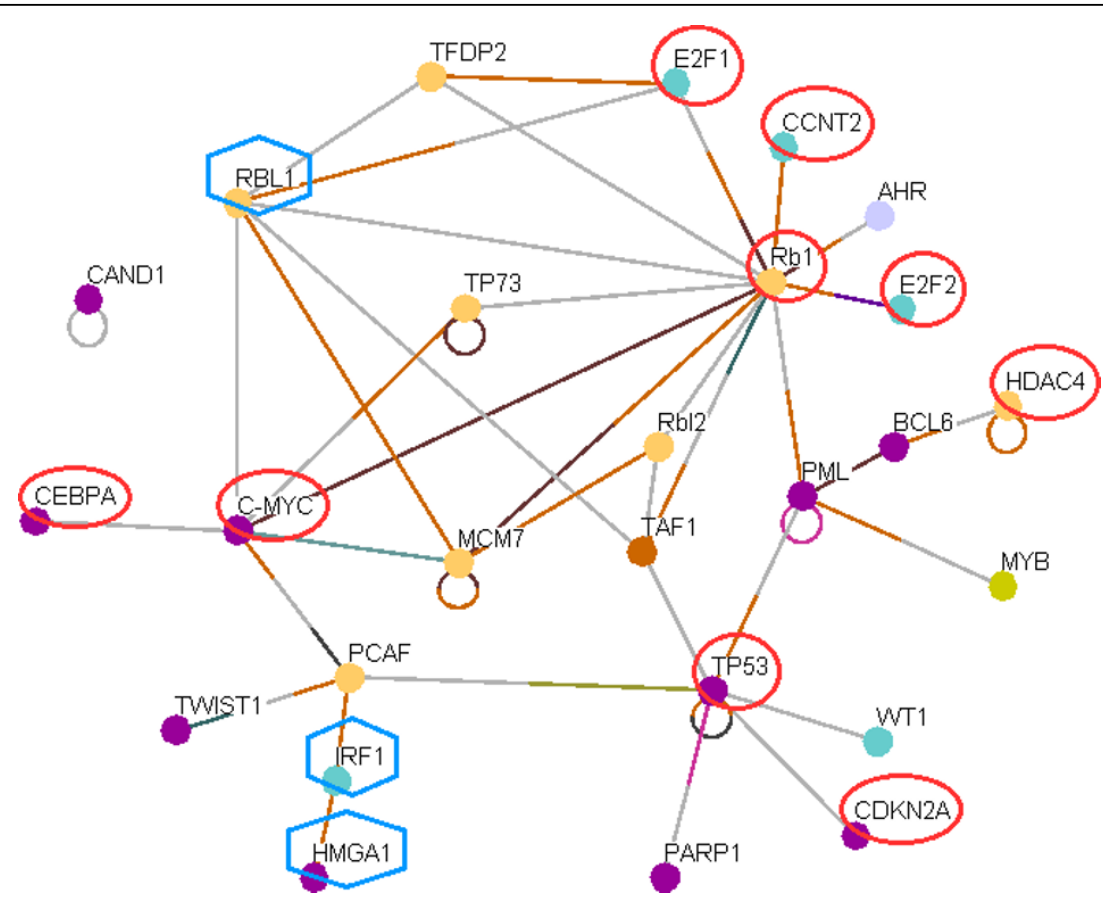

Figure 2 Cell cycle specific 26 key interacting TFs that are targets of miRNAs involved in common events in lung cancer as well as in NSCLC and SCLC. The network is created as described in the text. As per our hypothesis, this network also represents interactions of cell cycle regulating miRNAs associated with NSCLC, SCLC, and common events of lung cancer. TFs circled in red are shared by both NSCLC and SCLC. Molecules marked in hexagon are unique to common events. Other molecules in the map are shared by NSCLC and common events of lung cancers.

(2) general events, NSCLC, and SCLC; (3) NSCLC and general; (4) NSCLC specific; and (5) general events in lung cancers. The analysis revealed that nine genes (RB1, E2F1, E2F2, CCNT2, CMYC, CEBPA, TP53, CDKN2A, and HDAC4) that are key nodes in SCLC are common to

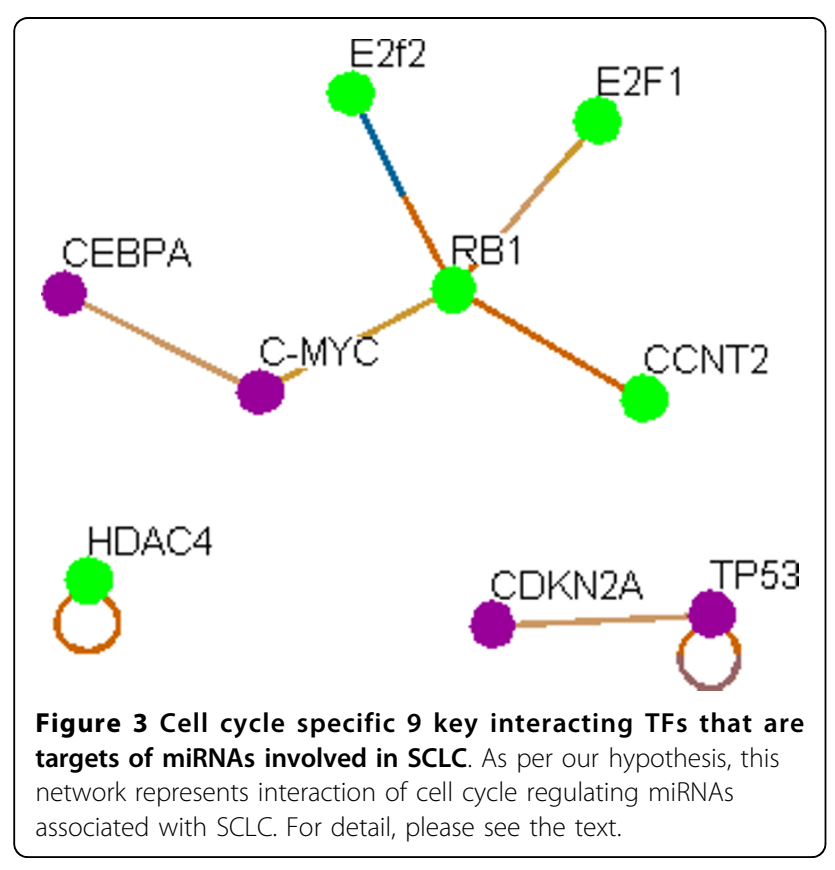

both the (1) SCLC and NSCLC and (2) general events, NSCLC, and SCLC groups (Table 1, group-1-3). Therefore, all of the SCLC genes are involved in NSCLC and in general events in lung cancer. Fourteen unique genes (Table 1, group-4) are found to be involved in both NSCLC and general events. The comparison also shows that four genes (Table 1, group-5) are specific to NSCLC and three genes (Table 1, group-6) are unique to general events. Therefore, these gene sets can be used in combination and their expression signature may be useful as diagnostic markers for NSCLC.

\section{Validation of markers}

We selected seven genes [4 unique genes (E2F6, TFDP1, SUV39H1, and HNRPD) for NSCLC and 3 genes (RBL1, IRF1, and HMGA1) for general events] for validation as diagnostic markers in lung cancer. Frozen NSCLC tissuebased microarray analysis revealed that E2F6, TFDP1, SUV39H1, and HMGA1 are significantly upregulated in both the adenocarcinoma and squamous cell carcinoma samples. The upregulation of RBL1 and downregulation of IRF1 in the microarray analysis was significant in squamous cell carcinoma but was statistically insignificant in adenocarcinoma (Additional file 6).

qPCR validation of markers based on blood samples showed expression patterns similar to the tissue based microarray analysis. TFPD1, E2F6, IRF1, and HMGA1 are 


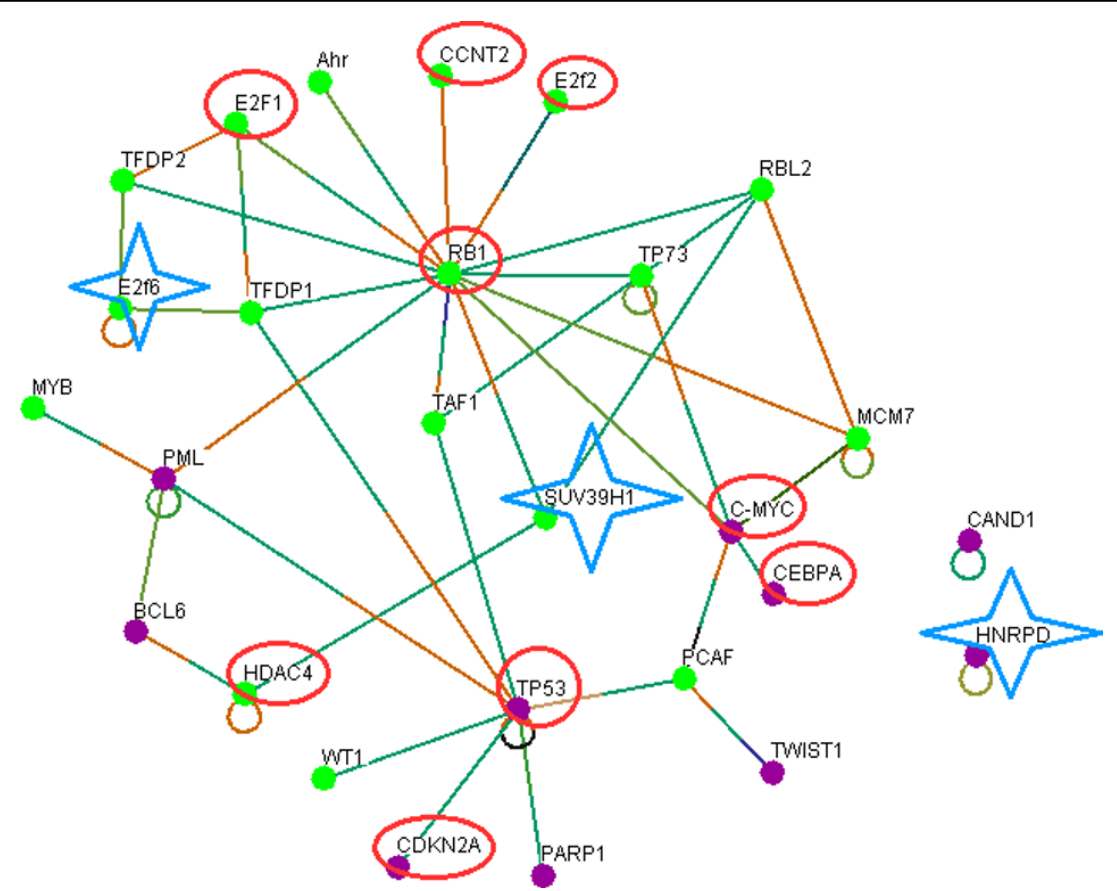

Figure 4 Interactions of TFs (as per our hypothesis miRNAs) associated with NSCLC and SCLC. TFs circled in red are shared by both NSCLC and SCLC. Molecules marked in star are unique to NSCLC. Other molecules in the map are shared by NSCLC and general events of lung cancers.

upregulated in all cancer samples. SUV39H1, RBL1, and HNRPD are downregulated or not expressed in all samples compared to the control (Figure 5). Therefore, combining the microarray and qPCR results, upregulation of E2F6, HMGA1, IRF1, and TFDP1 and downregulation or no expression of SUV39H1, RBL1, HNRPD can be used as diagnostic markers of NSCLC, and, in particular, adenocarcinoma and squamous cell carcinoma.

\section{Discussion}

In this work we have identified key transcription factors that can be useful biomarkers in diagnosis of lung cancer using an in silico reverse-transcriptomics approach. In this novel approach, starting with deregulated miRNAs in lung cancers we have identified transcription factors that can act as biomarkers, even for sub-type specific lung cancers. Out of several putative markers we identified,
7 NSCLC specific markers were validated. We found that E2F6, HMGA1, IRF1, and TFDP1 were upregulated and RBL1, SUV39H1, and HNRPD were downregulated or aberrantly expressed in adenocarcinoma and squamous cell carcinoma, which are the sub-types of NSCLC.

HMGA1 (High mobility group AT-hook 1) is an oncogene that is induced by Wnt/beta-catenin pathway and which positively regulates cell proliferation in gastric cancer [34]. By downregulating E-cadherin and upregulating expression of TWIST1, it enhances epithelial-mesenchymal transition and metastasis in colon cancer [35]. Upregulation of HMGA1 in glioblastoma positively correlates with malignancy, angiogenesis, and invasion [36]. In lung cancer, it is also overexpressed and increased nuclear expression correlates with poor survival in lung adenocarcinomas $[37,38]$. By upregulating PI3K and MMP2, it promotes cell migration and invasion $[37,39]$ and by

Table 1 Identified putative markers in lung cancers using the in silico reverse transcriptomics approach

\begin{tabular}{lll}
\hline Group & \multicolumn{1}{c}{ LC Types } \\
\hline 1 & Unique to SCLC & RB1, E2F1, E2F2, CCNT2, CMYC, CEBPA, TP53, CDKN2A, HDAC4 \\
2 & Common to SCLC and NSCLC & RB1, E2F1, E2F2, CCNT2, CMYC, CEBPA, TP53, CDKN2A, HDAC4 \\
3 & Common to general, SCLC, and NSCLC & RB1, E2F1, E2F2, CCNT2, CMYC, CEBPA, TP53, CDKN2A, HDAC4 \\
4 & Common to NSCLC and general & TFDP2, AHR, CCND1, TP73, RBL2, TAF1, PML, BCL6, MYB, WT1, PARP1, PCAF, TWIST, MCM7 \\
5 & NSCLC specific & E2F6, TFDP1, SUV39H1, HNRPD \\
6 & General/ common path specific & RBL1, IRF1, HMGA1 \\
\hline
\end{tabular}

The markers can be used in combination to design panels for diagnosis of sub-type specific lung cancers. 


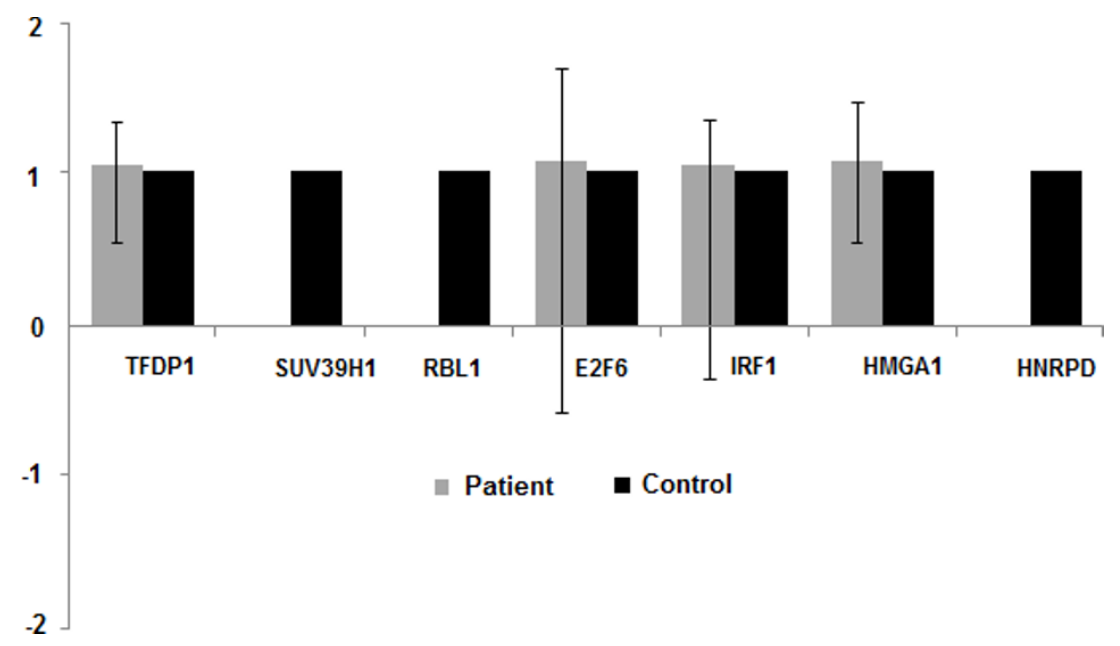

Figure 5 Blood based qPCR results for selected seven NSCLC specific markers. As compared to the control; HMGA1, TFPD1, E2F6, and IRF1 are upregulated and SUV39H1, RBL1, and HNRPD are downregulated or not expressed in all tested samples.

activating miR-222 oncomiR, it induces PPP2R2A mediated AKT signaling in NSCLC [40]. Therefore, upregulation of HMGA1 plays a significant role in tumor progression in NSCLC. In our study, we also observed that HMGA1 was upregulated in NSCLC supporting the previous findings.

TFDP1 (Transcription factor Dp-1) is a candidate oncogene that positively regulates S-phase entry and inhibits apoptosis in cooperation with E2F1 [41]. It is amplified and overexpressed in breast cancer [42] and upregulation of TFDP1 positively correlates with tumor size and progression of hepatocellular carcinomas [43] and increased cell viability in lung cancer [44]. In our observation, TFDP1 was overexpressed in all lung adenocarcinomas and squamous cell carcinomas, which supports the previous findings of Lu et al. (2000) in a SCLC cell line [45].

In our study, we observed IRF1 (Interferon regulatory factor 1) was upregulated in all NSCLC samples tested, although it had been shown to be downregulated in lung cancer in a previous study [46]. IRF1 inhibits G1-S cell cycle progression through P53 and p21 mediated pathways [46] and may act as a tumor-suppressor gene. This finding is supported by the findings that it is downregulated in gastric [47] and recurrent breast cancers [48]. However, IRF1 may not always act as a tumor-suppressor, as there is a report that it is upregulated in skin squamous cell carcinoma [49]. Therefore, our observation of upregulated IRF1 in NSCLC samples requires further attention to explore the precise role of this TF in various cancers.

E2F6 (E2F transcription factor 6) inhibits entry into S phase of cells stimulated to exit G0 [50] and inhibits apoptosis through E2F1 [51]. It may therefore play a role in cell proliferation and cell survival. There is no report about this protein's expression pattern in any cancer. Here, we have, for the first time, observed that E2F6 was upregulated in all of our tested NSCLC samples. This finding supports E2F6's putative role in tumorigenesis and shows that it may be a novel marker for NSCLC.

SUV39H1 (Suppressor of variegation 3-9 homolog 1) is a histone methyltransferase that inhibits inflammatory responses by downregulating interleukin- 6 production [52]. SUV39H1 inhibits the expression of CCND1 and may thereby negatively regulate cell proliferation [53]. However, its overexpression induces cell migration in breast and colon cancers [54] and negatively regulates apoptosis in a lung cancer model [55]. The expression level of SUV39H1 inversely correlates with stage, prognosis, and disease free survival in oral squamous cell carcinoma [56] and breast cancer [57]. Therefore, SUV39H1 may also have oncogenic properties. Although SUV39H1 was significantly upregulated in adenocarcinoma and squamous cell carcinoma tissue samples in our microarray analysis, supporting its positive role in tumorigenesis, it was found to be downregulated in blood samples in our qPCR validation. Therefore, SUV39H1 expression differs in lung cancer tissue and blood samples.

RBL1 (Retinoblastoma-like 1 (p107)) inhibits cell proliferation through G1 arrest [58] and positively regulates epidermal differentiation [59]. RBL1 is downregulated and inversely correlates with the histological grade of squamous cell carcinomas and adenocarcinomas [60]. Our qPCR validation shows downregulation in all squamous cell carcinoma and adenocarcinoma samples, which supports the previous findings and RBL1's function in tumors. 
HNRPD/AUF1 is a RNA-binding protein that both positively and negatively regulates neoplastic gene regulatory networks in cancer depending on the type of neoplasm [61]. It binds to destabilize p $21 \mathrm{mRNA}$ and thereby inhibits its anti-apoptotic activity [62]. Although in our blood-based qPCR analysis AUF1 was downregulated in all NSCLC samples, it has been reported to be upregulated in HCC [63] and experimental murine lung cancer [64]. It has been patented to aid in the prediction of survival in lung cancer in a gene expression panel of biomarkers (US 20100267574).

miRNA-markerTFs correlation: The seven identified TFs that are aberrantly expressed in both the squamous cell carcinoma and adenocarcinoma were plotted for their interactions with miRNAs and other key TFs to obtain more insight into these markers in lung cancer pathogenesis (Figure 1, Steps-14-15). The miRNA-TFCancer relationships were gathered from the miReg [17], miR2Disease [23], miRWalk [25], miRecords [26], TransmiR [31], CircuitsDB [32], and miRDB [65] databases. The interaction map is represented in Figure 6.
The network clearly shows meaningful relationships between the TFs and miRNAs in lung cancer. The interactions show that the tumor suppressor miRNAs (miR29a, miR-16, miR-125, and let-7) that could target the oncogene HMGA1 are downregulated. Upregulation of HMGA1 induces expression of oncogenic miR-122. Another two pro-oncogenic miRNAs that can also target HMGA1, miR-196a-2 and miR-155, are upregulated in lung cancers $[66,67]$. We observed that HMGA1 may inhibit the putative tumor-suppressor IRF1 (as per the interaction network) and that the miR-155 pro-oncomiR directly targeted IRF1. Therefore, in this network, HMGA1 is the key TF that positively regulates lung tumorigenesis through upregulation of miR-122 and perhaps by downregulation of IRF1. However, we found that IRF1 is upregulated in the samples so that the IRF1-HMGA1 interactions need further attention.

Tumor suppressor RBL1 is a target of the miR-17 oncomiR [68]. Furthermore, as per the interaction network, RBL1 is activated by TAF1 and cMYC, and regulates expression of E2F2, RB1, MCM7, and TFDP2.

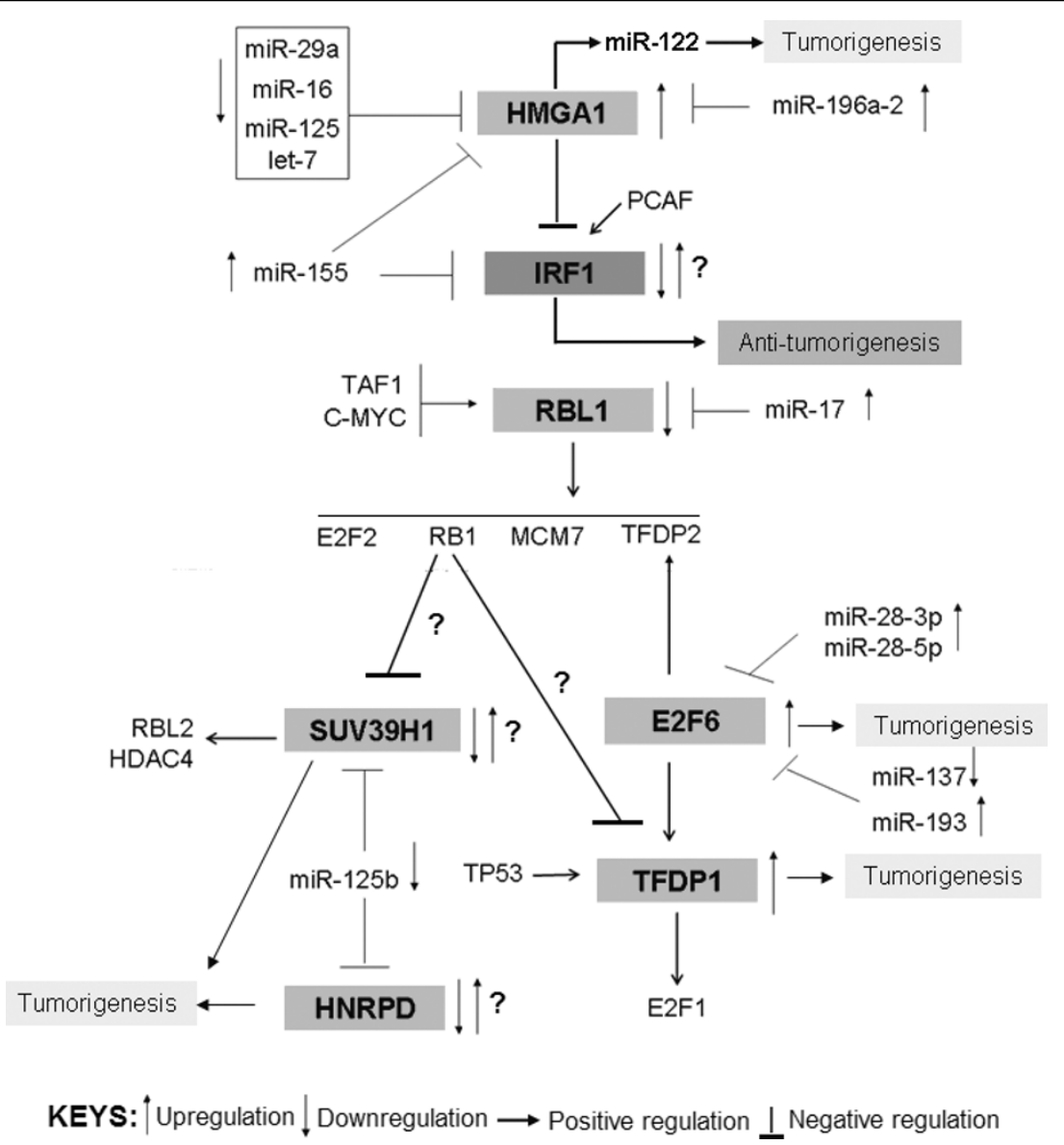

Figure 6 The correlations of identified seven TF markers and interacting miRNAs. The interactions provide better insights of molecular events and mechanisms during lung cancer tumorigenesis. For detail, please see the text. 
It thereby regulates the cell cycle and cell proliferation. Therefore, RBL1 downregulation and upregulation of miR-17 provide a meaningful mechanism in lung cancer tumorigenesis $[66,69]$.

The common pathway (of both NSCLC and SCLC) related genes HNRPD, E2F6, TFDP1, and SUV39H1 also showed the expected TF-miRNA relationship in the interaction map represented in Figure 6 based on the available experimental evidence. The literature shows that HNRPD and SUV39H1 may have positive roles in tumorigenesis $[55,56,64]$. Although in our blood-based qPCR, HNRPD and SUV39H1 are downregulated, they are reported to be upregulated in a mouse model of lung cancer [63], consistent with the tissue-based microarray analysis in our lung cancer samples. The involvement of HNRPD and SUV39H1 is further supported by reports that the tumor suppressor miR-125 is downregulated in both NSCLC and SCLC [70,71]. Furthermore, the tumor suppressor protein RB1 is downregulated in lung cancer [66] and may inhibit SUV39H1.

The other two markers, E2F6 and TFDP1, are upregulated in all of our blood samples. While two pro-oncogenic miRNAs, miR-28 and miR-193, are upregulated [40] the putative tumor-suppressor, miR-137, is downregulated in lung cancers $[72,73]$. All three of these miRNAs target E2F6 $[74,75]$. Furthermore, E2F6 putatively upregulates TFDP1 and is downregulated by RB1. It is also found from the interaction map that E2F6 inhibition by two upregulated pro-oncomiRs (miR-28 and miR-193) is not sufficient, as the E2F6 was found to be upregulated in lung cancer. Further, E2F6 has been reported to upregulate oncogene TFDP1 and to positively regulate cell proliferation and cell survival through E2F1 [41]. Additionally, downregulation of RB1 in lung cancer is not able to repress TFDP1 activity, and therefore, in lung cancer, tumorigenesis is mediated through upregulation of E2F6 and TFDP1. However, the role of SUV39H1 and HNRPD requires further exploration.

\section{Conclusion}

In this analysis, using an integrated reverse-transcriptomics-based bioinformatics approach, we have identified key transcription factors that may be useful in developing subtype specific biomarkers in lung cancer. Our proposed seven markers also have high potential to be used in lung cancer diagnostics for NSCLC subtypes. Of course, additional experimental validation in independent sets of patients is required to establish the diagnostic accuracy of this panel and we are currently conducting those experiments. The miRNA-TF-miRNA relationships with these seven miRNAs show meaningful associations with these TFs in lung cancer pathogenesis. The novel strategy developed in this research is powerful and can be applicable to identify molecular mechanisms and markers in other cancers as well.

\section{Funding}

This work was carried out without any grant. VA had funding from CNPq and FAPEMIG.

\section{Additional material}

Additional file 1: List of primers to amplify TFDP1, SUV39H1, RBL1,
E2FG, IRF1, HMGA1, and HNRPD.
Additional file 2: Common miRNAs involved in both NSCLC and
SCLC. The differentially expressed miRNAs are marked with blue.
Additional file 3: Small-cell-lung cancer (SCLC) specific 22
deregulated miRNAs (16 upregulated and 6 downregulated).
Additional file 4: Non-small-cell lung cancer (NSCLC) specific 143
deregulated miRNAs (89 upregulated and 43 downregulated). The
miRNAs that are reported upregulated in one report but downregulated
in other report or vise versa are highlighted in blue.
Additional file 5: Functional annotation of common miRNAs using
the targets of these miRNAs and DAVID
Additional file 6: Microarray based expression analysis of NSCLC
specific 6 identified markers [E2F6, TFDP1, and SUV39H1 for NSCLC
and RBL1, IRF1, and HMGA1 for general events]. E2F6, TFDP1,
SUV39H1, and HMGA1 are significantly upregulated in both the
adenocarcinoma and squamous cell carcinoma samples. The
upregulation of RBL1 and downregulation of IRF1 in the microarray
analysis was significant in squamous cell carcinoma but was statistically
insignificant in adenocarcinoma.

Conflict of interest

Authors declare no conflict of interest.

\section{Authors' contributions}

DB: Conceived the idea, designed the study, coordinated and leaded the entire project, and wrote the manuscript; $\mathbf{D B}, \mathbf{N J}$ : collected and analyzed primary data, DB, NJ, ST, AB, LJ: performed all in silico analyses; JKF, TL: performed microarray analysis; EP, AR, RL, MH: performed QPCR experiments; PG, CV, AK, AS, AM, VA, KB: cross verified all analyses. All authors have read and approved the manuscript.

\section{Declarations}

Publication for this article has been funded by NSF 1158608. This article has been published as part of BMC Genomics Volume 14 Supplement 6, 2013: Proceedings of the International Conference of the Brazilian Association for Bioinformatics and Computational Biology (X-meeting 2012). The full contents of the supplement are available online at http://www.biomedcentral.com/bmcgenomics/supplements/14/S6.

\section{Authors' details}

${ }^{1}$ Centre for Genomics and Applied Gene Technology, Institute of Integrative Omics and Applied Biotechnology (IIOAB), Nonakuri, Purba Medinipur, WB721172, India. ${ }^{2}$ School of Biotechnology, Devi Ahilya University, Khandwa Road Campus, Indore, MP, India. ${ }^{3}$ University of Liverpool, Department of Molecular and Clinical Cancer Medicine, 200 London Road, Liverpool L3 9TA, UK. ${ }^{4}$ Medical Oncology Department, Complejo Hospitalario Universitario, Santiago de Compostela, A Coruña, Spain. ${ }^{5}$ Nuclear Medicine Service, Complejo Hospitalario Universitario. Fundación Tejerina. Santiago de Compostela, A Coruña, Spain. ${ }^{6}$ Molecular Oncology and Imaging Program, Complejo Hospitalario Universitario, Santiago de Compostela, A Coruña, Spain. ${ }^{7}$ Institute of Molecular and Experimental Medicine, Cardiff University, Cardiff CF14 4XN, Wales, UK. ${ }^{8}$ Instituto de Ciências Biológicas, Universidade Federal do Pará, Rua Augusto Corrêa, 01 - Guamá, Belém, PA, Brazil. 
${ }^{9}$ Laboratorio de Genetica Celular e Molecular, Departmento de Biologia Geral, Instituto de Ciencias Biologics, Universidade Federal de Minas Gerais CP 486, CEP 31270-901 Belo Horizonte, Minas Gerais, Brazil. ${ }^{10}$ Department of Psychiatry and Mcknight Brain Institute, College of Medicine, University of Florida, University Ave., Gainesville, FL 32601, USA. ${ }^{1}$ Department of Computer Science and Center for the Study of Biological Complexity, Virginia Commonwealth University, Richmond, Virginia, USA.

Published: 25 October 2013

\section{References}

1. Jemal A, Bray F, Center MM, Ferlay J, Ward E, Forman D: Global cancer statistics. CA Cancer J Clin 2011, 61(2):69-90.

2. Petersen I: The morphological and molecular diagnosis of lung cancer. Dtsch Arztebl Int 2011, 108(31-32):525-531.

3. Wang T, Nelson RA, Bogardus A, Grannis FW Jr: Five-year lung cancer survival: which advanced stage nonsmall cell lung cancer patients attain long-term survival? Cancer 2010, 116(6):1518-1525.

4. Pastorino U: Lung cancer screening. Br J Cancer 2010, 102(12):1681-1686.

5. Granville CA, Dennis PA: An overview of lung cancer genomics and proteomics. Am J Respir Cell Mol Biol 2005, 32(3):169-176.

6. Bianchi F, Nicassio F, Marzi M, Belloni E, Dall'olio V, Bernard L, Pelosi G, Maisonneuve P, Veronesi G, Di Fiore PP: A serum circulating miRNA diagnostic test to identify asymptomatic high-risk individuals with early stage lung cancer. EMBO Mol Med 2011, 3(8):495-503.

7. Hennessey PT, Sanford T, Choudhary A, Mydlarz WW, Brown D, Adai AT, Ochs MF, Ahrendt SA, Mambo E, Califano JA: Serum microRNA Biomarkers for Detection of Non-Small Cell Lung Cancer. PLoS One 2012, 7(2):e32307.

8. Yu L, Todd NW, Xing L, Xie Y, Zhang H, Liu Z, Fang H, Zhang J, Katz RL, Jiang F: Early detection of lung adenocarcinoma in sputum by a panel of microRNA markers. Int J Cancer 2010, 127(12):2870-2878.

9. Liao J, Yu L, Mei Y, Guarnera M, Shen J, Li R, Liu Z, Jiang F: Small nucleolar RNA signatures as biomarkers for non-small-cell lung cancer. Mol Cancer 2010, 9:198.

10. Xing L, Todd NW, Yu L, Fang H, Jiang F: Early detection of squamous cell lung cancer in sputum by a panel of microRNA markers. Mod Pathol 2010, 23(8):1157-1164.

11. Guergova-Kuras M, Kurucz I, Hempel W, Tardieu N, Kádas J, MalderezBloes C, Jullien A, Kieffer Y, Hincapie M, Guttman A, Csánky E, Dezso B, Karger BL, Takács L: Discovery of lung cancer biomarkers by profiling the plasma proteome with monoclonal antibody libraries. Mol Cell Proteomics 2011, 10(12):M111.010298.

12. Tong BC, Harpole DH Jr: Molecular markers for incidence, prognosis, and response to therapy. Surg Oncol Clin N Am 2012, 21(1):161-75.

13. Xiao H, Zhang L, Zhou H, Lee JM, Garon EB, Wong DT: Proteomic analysis of human saliva from lung cancer patients using two-dimensional difference gel electrophoresis and mass spectrometry. Mol Cell Proteomics 2012, 11(2):M111.012112.

14. Begum S, Brait M, Dasgupta S, Ostrow KL, Zahurak M, Carvalho AL, Califano JA, Goodman SN, Westra WH, Hoque MO, Sidransky D: An epigenetic marker panel for detection of lung cancer using cell-free serum DNA. Clin Cancer Res 2011, 17(13):4494-4503.

15. Jiang F, Todd NW, Li R, Zhang H, Fang H, Stass SA: A panel of sputumbased genomic marker for early detection of lung cancer. Cancer Prev Res (Phila) 2010, 3(12):1571-1578.

16. Showe MK, Vachani A, Kossenkov AV, Yousef M, Nichols C, Nikonova EV, Chang C, Kucharczuk J, Tran B, Wakeam E, Yie TA, Speicher D, Rom WN, Albelda S, Showe LC: Gene expression profiles in peripheral blood mononuclear cells can distinguish patients with non-small cell lung cancer from patients with nonmalignant lung disease. Cancer Res 2009, 69(24):9202-9210.

17. Barh $D$, Bhat $D$, Viero $C$ : miReg: a resource for microRNA regulation. J Integr Bioinform 2010, 7(1):144.

18. Blenkiron C, Miska EA: miRNAs in cancer: approaches, aetiology, diagnostics and therapy. Hum Mol Genet 2007, 16(1):R106-13.

19. Sempere LF, Liu X, Dmitrovsky E: Tumor-suppressive microRNAs in Lung cancer: diagnostic and therapeutic opportunities. Scientific World Journal 2009, 9:626-628.

20. Barshack I, Lithwick-Yanai G, Afek A, Rosenblatt K, Tabibian-Keissar H, Zepeniuk M, Cohen L, Dan H, Zion O, Strenov Y, Polak-Charcon S,
Perelman M: MicroRNA expression differentiates between primary lung tumors and metastases to the lung. Pathol Res Pract 2010, 206(8):578-584.

21. Hu Z, Chen X, Zhao Y, Tian T, Jin G, Shu Y, Chen Y, Xu L, Zen K, Zhang C, Shen $\mathrm{H}$ : Serum microRNA signatures identified in a genome-wide serum microRNA expression profiling predict survival of non-small-cell lung cancer. J Clin Oncol 2010, 28(10):1721-1726.

22. Esquela-Kerscher A, Slack FJ: Oncomirs - microRNAs with a role in cancer. Nat Rev Cancer 2006, 6(4):259-269.

23. Jiang $Q$, Wang $Y$, Hao $Y$, Juan L, Teng M, Zhang $X$, Li M, Wang G, Liu Y: miR2Disease: a manually curated database for microRNA deregulation in human disease. Nucleic Acids Res 2009, 37(Database):D98-104.

24. Tsou JA, Galler JS, Siegmund KD, Laird PW, Turla S, Cozen W, Hagen JA, Koss MN, Laird-Offringa IA: Identification of a panel of sensitive and specific DNA methylation markers for lung adenocarcinoma. Mol Cancer 2007, 6:70.

25. Dweep H, Sticht C, Pandey P, Gretz N: miRWalk-database: prediction of possible miRNA binding sites by "walking" the genes of three genomes. J Biomed Inform 2011, 44(5):839-47.

26. Xiao F, Zuo Z, Cai G, Kang S, Gao X, Li T: miRecords: an integrated resource for microRNA-target interactions. Nucleic Acids Res 2009, 37(Database):D105-10.

27. Hsu SD, Lin FM, Wu WY, Liang C, Huang WC, Chan WL, Tsai WT, Chen GZ, Lee CJ, Chiu CM, Chien CH, Wu MC, Huang CY, Tsou AP, Huang HD: miRTarBase: a database curates experimentally validated microRNAtarget interactions. Nucleic Acids Res 2011, 39(Database):D163-9.

28. Chen J, Bardes EE, Aronow BJ, Jegga AG: ToppGene Suite for gene list enrichment analysis and candidate gene prioritization. Nucleic Acids Res 2009, 37(Web Server):W305-11.

29. Huang DW, Sherman BT, Lempicki RA: Systematic and integrative analysis of large gene lists using DAVID Bioinformatics Resources. Nature Protoc 2009, 4(1):44-57.

30. Breitkreutz BJ, Stark C, Tyers M: Osprey: a network visualization system. Genome Biol 2003, 4(3):R22.

31. Wang J, Lu M, Qiu C, Cui Q: TransmiR: a transcription factor-microRNA regulation database. Nucleic Acids Res 2010, 38(Database):D119-22.

32. Friard O, Re A, Taverna D, De Bortoli M, Corá D: CircuitsDB: a database of mixed microRNA/transcription factor feed-forward regulatory circuits in human and mouse. BMC Bioinformatics 2010, 11:435.

33. Hu Z, Hung JH, Wang Y, Chang YC, Huang CL, Huyck M, DeLisi C: VisANT 3.5: multi-scale network visualization, analysis and inference based on the gene ontology. Nucleic Acids Res 2009, 37(Web Server):W115-21.

34. Akaboshi $S$, Watanabe $S$, Hino $Y$, Sekita $Y, X i$, Araki K, Yamamura $K$, Oshima M, Ito T, Baba H, Nakao M: HMGA1 is induced by Wnt/betacatenin pathway and maintains cell proliferation in gastric cancer. Am J Pathol 2009, 175(4):1675-1685.

35. Belton A, Gabrovsky A, Bae YK, Reeves R, lacobuzio-Donahue C, Huso DL, Resar LM: HMGA1 induces intestinal polyposis in transgenic mice and drives tumor progression and stem cell properties in colon cancer cells. PLoS One 2012, 7(1):e30034.

36. Pang B, Fan H, Zhang IY, Liu B, Feng B, Meng L, Zhang R, Sadeghi S, Guo H, Pang Q: HMGA1 expression in human gliomas and its correlation with tumor proliferation, invasion and angiogenesis. J Neurooncol 2012, 106(3):543-549.

37. Hillion J, Wood L, Mukherjee M, Bhattacharya R, Di Cello F, Kowalski J, Elbahloul O, Segal J, Poirier J, Rudin CM, Dhara S, Belton A, Joseph B, Zucker S, Resar LM: Upregulation of MMP-2 by HMGA1 promotes transformation in undifferentiated, large-cell lung cancer. Mol Cancer Res 2009, 7(11):1803-1812.

38. Sarhadi VK, Wikman H, Salmenkivi K, Kuosma E, Sioris T, Salo J, Karjalainen A, Knuutila S, Anttila S: Increased expression of high mobility group A proteins in lung cancer. J Pathol 2006, 209(2):206-212.

39. Scrima M, De Marco C, Fabiani F, Franco R, Pirozzi G, Rocco G, Ravo M, Weisz A, Zoppoli P, Ceccarelli M, Botti G, Malanga D, Viglietto G: Signaling networks associated with AKT activation in non-small cell lung cancer (NSCLC): new insights on the role of phosphatydil-inositol-3 kinase. PLOS One 2012, 7(2):e30427.

40. Zhang Y, Ma T, Yang S, Xia M, Xu J, An H, Yang Y, Li S: High-mobility group A1 proteins enhance the expression of the oncogenic miR-222 in lung cancer cells. Mol Cell Biochem 2011, 357(1-2):363-371.

41. Shan B, Farmer AA, Lee WH: The molecular basis of E2F-1/DP-1-induced S-phase entry and apoptosis. Cell Growth Differ 1996, 7(6):689-697. 
42. Abba MC, Fabris VT, Hu Y, Kittrell FS, Cai WW, Donehower LA, Sahin A Medina D, Aldaz CM: Identification of novel amplification gene targets in mouse and human breast cancer at a syntenic cluster mapping to mouse ch8A1 and human ch13q34. Cancer Res 2007, 67(9):4104-4112.

43. Yasui $\mathrm{K}$, Okamoto $\mathrm{H}$, Arii $\mathrm{S}$, Inazawa J: Association of over-expressed TFDP1 with progression of hepatocellular carcinomas. J Hum Genet 2003, 48(12):609-613.

44. Castillo SD, Angulo B, Suarez-Gauthier A, Melchor L, Medina PP, SanchezVerde L, Torres-Lanzas J, Pita G, Benitez J, Sanchez-Cespedes M: Gene amplification of the transcription factor DP1 and CTNND1 in human lung cancer. J Pathol 2010, 222(1):89-98.

45. Lu K, Shih C, Teicher BA: Expression of pRB, cyclin/cyclin-dependent kinases and E2F1/DP-1 in human tumor lines in cell culture and in xenograft tissues and response to cell cycle agents. Cancer Chemother Pharmacol 2000, 46(4):293-304.

46. Usuda J, Saijo N, Fukuoka K, Fukumoto H, Kuh HJ, Nakamura T, Koh Y, Suzuki T, Koizumi F, Tamura T, Kato H, Nishio K: Molecular determinants of UCN-01-induced growth inhibition in human lung cancer cells. Int J Cancer 2000, 85(2):275-280.

47. Nozawa H, Oda E, Ueda S, Tamura G, Maesawa C, Muto T, Taniguchi T, Tanaka N: Functionally inactivating point mutation in the tumorsuppressor IRF-1 gene identified in human gastric cancer. Int J Cancer 1998, 77(4):522-7.

48. Cavalli LR, Riggins RB, Wang A, Clarke R, Haddad BR: Frequent loss of heterozygosity at the interferon regulatory factor- 1 gene locus in breast cancer. Breast Cancer Res Treat 2010, 121(1):227-231.

49. Wenzel J, Tomiuk S, Zahn S, Küsters D, Vahsen A, Wiechert A, Mikus S, Birth M, Scheler M, von Bubnoff D, Baron JM, Merk HF, Mauch C, Krieg T, Bieber T, Bosio A, Hofmann K, Tüting T, Peters B: Transcriptional profiling identifies an interferon-associated host immune response in invasive squamous cell carcinoma of the skin. Int J Cancer 2008, 123(11):2605-15.

50. Gaubatz S, Wood JG, Livingston DM: Unusual proliferation arrest and transcriptional control properties of a newly discovered E2F family member, E2F-6. Proc Natl Acad Sci USA 1998, 95(16):9190-9195.

51. Yang WW, Shu B, Zhu Y, Yang HT: E2F6 inhibits cobalt chloride-mimetic hypoxia-induced apoptosis through E2F1. Mol Biol Cell 2008, 19(9):3691-3700

52. Villeneuve $L M$, Reddy MA, Lanting LL, Wang M, Meng L, Natarajan R: Epigenetic histone $\mathrm{H} 3$ lysine 9 methylation in metabolic memory and inflammatory phenotype of vascular smooth muscle cells in diabetes. Proc Natl Acad Sci USA 2008, 105(26):9047-9052.

53. Yang YJ, Han JW, Youn HD, Cho EJ: The tumor suppressor, parafibromin mediates histone $\mathrm{H} 3 \mathrm{~K} 9$ methylation for cyclin D1 repression. Nucleic Acids Res 2010, 38(2):382-90.

54. Yokoyama $Y$, Hieda M, Nishioka $Y$, Matsumoto A, Higashi S, Kimura H, Yamamoto H, Mori M, Matsuura S, Matsuura N: Cancer associated upregulation of $\mathrm{H} 3 \mathrm{~K} 9$ trimethylation promotes cell motility in vitro and drives tumor formation in vivo. Cancer Sci 2013, doi: 10.1111/cas.12166.

55. Watanabe H, Soejima K, Yasuda H, Kawada I, Nakachi I, Yoda S, Naoki K, Ishizaka A: Deregulation of histone lysine methyltransferases contributes to oncogenic transformation of human bronchoepithelial cells. Cancer Cell Int 2008, 8:15.

56. Chen JH, Yeh KT, Yang YM, Chang JG, Lee HE, Hung SY: High expressions of histone methylation- and phosphorylation-related proteins are associated with prognosis of oral squamous cell carcinoma in male population of Taiwan. Med Oncol 2013, 30(2):513.

57. Patani N, Jiang WG, Newbold RF, Mokbel K: Histone-modifier gene expression profiles are associated with pathological and clinical outcomes in human breast cancer. Anticancer Res 2011, 31(12):4115-25.

58. Zhu L, van den Heuvel S, Helin K, Fattaey A, Ewen M, Livingston D, Dyson N, Harlow E: Inhibition of cell proliferation by p107, a relative of the retinoblastoma protein. Genes Dev 1993, 7(7A):1111-1125.

59. Paramio JM, Laín S, Segrelles C, Lane EB, Jorcano JL: Differential expression and functionally co-operative roles for the retinoblastoma family of proteins in epidermal differentiation. Oncogene 1998, 17(8):949-957.

60. Baldi A, Esposito V, De Luca A, Howard CM, Mazzarella G, Baldi F, Caputi M, Giordano A: Differential expression of the retinoblastoma gene family members pRb/p105, p107, and pRb2/p130 in lung cancer. Clin Cancer Res 1996, 2(7):1239-1245.
61. Zucconi BE, Wilson GM: Modulation of neoplastic gene regulatory pathways by the RNA-binding factor AUF1. Front Biosci 2011, 16:2307-2325

62. Shchors K, Yehiely F, Kular RK, Kotlo KU, Brewer G, Deiss LP: Cell death inhibiting RNA (CDIR) derived from a 3'-untranslated region binds AUF1 and heat shock protein 27. J Biol Chem 2002, 277(49):47061-47072.

63. Frau M, Tomasi ML, Simile MM, Demartis MI, Salis F, Latte G, Calvisi DF, Seddaiu MA, Daino L, Feo CF, Brozzetti S, Solinas G, Yamashita S, Ushijima T, Feo F, Pascale RM: Role of transcriptional and posttranscriptional regulation of methionine adenosyltransferases in liver cancer progression. Hepatology 2012, 56(1):165-175.

64. Blaxall BC, Dwyer-Nield LD, Bauer AK, Bohlmeyer TJ, Malkinson AM, Port JD: Differential expression and localization of the mRNA binding proteins, AU-rich element mRNA binding protein (AUF1) and Hu antigen R (HuR), in neoplastic lung tissue. Mol Carcinog 2000, 28(2):76-83.

65. Xiaowei W: miRDB: a microRNA target prediction and functional annotation database with a wiki interface. RNA 2008, 14(6):1012-1017.

66. Volinia S, Calin GA, Liu CG, Ambs S, Cimmino A, Petrocca F, Visone R, lorio M, Roldo C, Ferracin M, Prueitt RL, Yanaihara N, Lanza G, Scarpa A, Vecchione A, Negrini M, Harris CC, Croce CM: A microRNA expression signature of human solid tumors defines cancer gene targets. Proc Natl Acad Sci USA 2006, 103(7):2257-2261.

67. Yanaihara N, Caplen N, Bowman E, Seike M, Kumamoto K, Yi M, Stephens RM, Okamoto A, Yokota J, Tanaka T, Calin GA, Liu CG, Croce CM, Harris CC: Unique microRNA molecular profiles in lung cancer diagnosis and prognosis. Cancer Cell 2006, 9(3):189-198.

68. Trompeter $\mathrm{HI}$, Abbad H, Iwaniuk KM, Hafner M, Renwick N, Tuschl T, Schira J, Müller HW, Wernet P: MicroRNAs MiR-17, MiR-20a, and MiR-106b act in concert to modulate E2F activity on cell cycle arrest during neuronal lineage differentiation of USSC. PLOS One 2011, 6(1):e16138.

69. Hayashita $Y$, Osada H, Tatematsu Y, Yamada H, Yanagisawa $K$, Tomida S, Yatabe $Y$, Kawahara $K$, Sekido $Y$, Takahashi T: A polycistronic microRNA cluster, miR-17-92, is overexpressed in human lung cancers and enhances cell proliferation. Cancer Res 2005, 65(21):9628-9632.

70. Miko E, Czimmerer Z, Csánky E, Boros G, Buslig J, Dezso B, Scholtz B: Differentially expressed microRNAs in small cell lung cancer. Exp Lung Res 2009, 35(8):646-664.

71. Raponi M, Dossey L, Jatkoe T, Wu X, Chen G, Fan H, Beer DG: MicroRNA classifiers for predicting prognosis of squamous cell lung cancer. Cancer Res 2009, 69(14):5776-5783.

72. Yu SL, Chen HY, Chang GC, Chen CY, Chen HW, Singh S, Cheng CL, Yu CJ, Lee YC, Chen HS, Su TJ, Chiang CC, Li HN, Hong QS, Su HY, Chen CC, Chen WJ, Liu CC, Chan WK, Chen WJ, Li KC, Chen JJ, Yang PC: MicroRNA signature predicts survival and relapse in lung cancer. Cancer Cell 2008, 13(1):48-57.

73. Dacic S, Kelly L, Shuai Y, Nikiforova MN: miRNA expression profiling of lung adenocarcinomas: correlation with mutational status. Mod Pathol 2010, 23(12):1577-1582

74. Girardot M, Pecquet C, Boukour S, Knoops L, Ferrant A, Vainchenker W, Giraudier S, Constantinescu SN: miR-28 is a thrombopoietin receptor targeting microRNA detected in a fraction of myeloproliferative neoplasm patient platelets. Blood 2010, 116(3):437-445.

75. Kozaki K, Imoto I, Mogi S, Omura K, Inazawa J: Exploration of tumorsuppressive microRNAs silenced by DNA hypermethylation in oral cancer. Cancer Res 2008, 68(7):2094-2105.

doi:10.1186/1471-2164-14-S6-S5

Cite this article as: Barh et al:: A novel in silico reverse-transcriptomicsbased identification and blood-based validation of a panel of sub-type specific biomarkers in lung cancer. BMC Genomics 2013 14(Suppl 6):S5. 